\title{
Hydroxy-tyrosol as a Free Radical Scavenging Molecule in Polymeric Hydrogels Subjected to Gamma-Ray Irradiation
}

\author{
Mauro Fiorini ${ }^{1,2, *}$, Veronica Crognaletti ${ }^{3}$, Omar Sabry ${ }^{2, *} \mathbb{C}$, Lorenzo Scalise ${ }^{3}$ and Paolo Fattori ${ }^{1,2}$ \\ 1 Sterify srl, 10126 Torino, Italy; p.fattori@sterify.it \\ 2 Tiss'You srl, 47895 Domagnano, San Marino \\ 3 Dipartimento di Ingegneria Industriale e Scienze Matematiche, Università Politecnica delle Marche, \\ 60131 Ancona, Italy; veronica.crognaletti.11@gmail.com (V.C.); 1.scalise@staff.univpm.it (L.S.) \\ * Correspondence: m.fiorini@sterify.it (M.F.); o.sabry@tissyou.com (O.S.)
}

Citation: Fiorini, M.; Crognaletti, V.; Sabry, O.; Scalise, L.; Fattori, P.

Hydroxy-tyrosol as a Free Radical Scavenging Molecule in Polymeric Hydrogels Subjected to Gamma-Ray Irradiation. Processes 2021, 9, 433. https://doi.org/10.3390/pr9030433

Academic Editor:

Shreyas Kuddannaya

Received: 26 November 2020

Accepted: 26 February 2021

Published: 27 February 2021

Publisher's Note: MDPI stays neutral with regard to jurisdictional claims in published maps and institutional affiliations.

Copyright: (c) 2021 by the authors. Licensee MDPI, Basel, Switzerland. This article is an open access article distributed under the terms and conditions of the Creative Commons Attribution (CC BY) license (https:/ / creativecommons.org/licenses/by/ $4.0 /)$.

\begin{abstract}
Biomedical engineering is employing hydrogels with increasingly exciting possibilities for the treatment and regeneration of pathologically altered, degenerated, or traumatized tissues. Still, the sterilization processes may undesirably change the chemical and physical properties of hydrogels through cross-linking reactions. This work aims to characterize a new method of producing polyethylene oxide (PEO) hydrogels exploiting hydroxy-tyrosol (HT), an anti-oxidant molecule derived from olive leaf and olive oil, as a free radical scavenger to either prevent or limit gamma-rayinduced cross-linking. For this purpose, we produced hydrogels with PEO with two different buffer solutions (phosphate and citrate), varying HT concentration. We analyzed hydrogel preparations before and after gamma-ray irradiation, assessing the viscosity through rheological analysis and the chemical changes through IR analysis. We performed high-performance liquid chromatography (HPLC) analysis to measure residual HT in hydrogels after irradiation. The obtained results show that radiation-induced cross-linking and increase in viscosity of PEO hydrogels can be prevented by tailoring the concentration of $\mathrm{HT}$ as a free radical scavenging agent. Irradiation only consumes small amounts of HT; its presence in polymeric hydrogels can significantly impact biomedical applications by its anti-oxidant and anti-microbial activities.
\end{abstract}

Keywords: hydroxy-tyrosol; hydrogel; irradiation-induced cross-linking; polyethylene oxide-based hydrogel; citrate buffer hydrogel; phosphate buffer hydrogel

\section{Introduction}

Hydrogels are three-dimensional polymeric networks with the ability to hold water within a porous structure. Thanks to their biocompatibility and mechanical properties, hydrogels are ideal scaffolds for tissue engineering, regenerative medicine, and drug delivery applications [1,2]. With this significant relevance in the biomedical field, sterilization of hydrogels is a conditio sine qua non to avoid any pathogen contamination risk and to fulfill medical device production requirements. However, sterilization may alter the structure and the characteristics of hydrogels inducing changes in the chemical and physical properties. Specifically, irradiation can cause cross-linking and polymerization reactions in conventional hydrogels, i.e., polyvinyl acetate (PVA), polyvinyl pyrrolidone (PVP), and polyethylene oxide (PEO) based synthetic formulations. As a consequence, these hydrogels undergo undesirable effects such as embrittlement, solidification, plasticization, or viscosity increase [3].

Compounds with anti-oxidant and free radical scavenging properties have been explored to limit cross-linking phenomena. Vitamin C can prevent irradiation-induced cross-linking of PVA solutions and decrease the viscosity of irradiated hydrogels, thus enhancing the stability and injectability of PVA-based medical formulations [4]. Vitamin E reduces cross-linking efficiency in ultrahigh molecular weight polyethylene during 
irradiation with the possibility of controlling the cross-link density distribution by tailoring the Vitamin E concentration profile [5].

Hydroxy-tyrosol (HT) is a phenylethanoid derived from olive leaf and olive oil with an extreme anti-oxidant efficiency, higher than Vitamin C [6]. Its high anti-oxidant activity is due to its free radical scavenging activity, attributed to the presence of the $o$-dihydroxyphenyl moiety which can release protons and promotes structural conjugation with radical species (Scheme 1) [7,8]. Notably, HT can inhibit low-density lipoprotein peroxidation chain reaction, which is a critical step in the development of atherosclerosis [9]. Moreover, HT shows biological impact against cancer development, osteoporosis, inflammation, bacteria proliferation, and virus infection $[10,11]$.

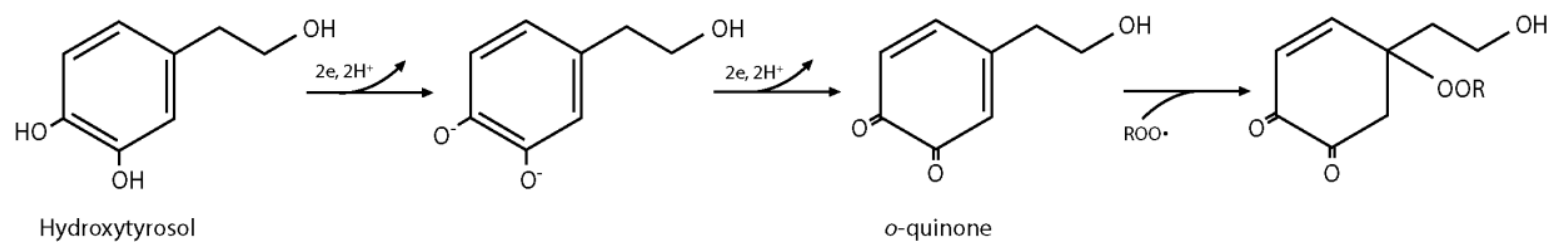

Scheme 1. Mechanism of free radical scavenging by hydroxy-tyrosol.

On the basis of these considerations, we hypothesized that HT could be a molecule of interest in preventing or limiting irradiation-induced cross-linking of hydrogels. This work aims to characterize the rheological and chemical properties after gamma-ray irradiation of both citrate and phosphate buffer PEO-based hydrogel with tailored concentration of HT.

\section{Materials and Methods}

\subsection{Hydrogels Preparation}

For the production of hydrogels, PEO $600 \mathrm{kDa}, 4 \% w / w(182028$, Sigma-Aldrich, St. Louis, MO, USA) was mixed with either Phosphate Buffer Solution or Citrate Buffer Solution. Phosphate Buffer Solution was bought ready to use (PBS; pH 6.00 1X; TL1102, HiMedia, Mumbai, India) and $1 \mathrm{~L}$ of $10 \mathrm{mM}$ of Citrate Buffer Solution has been prepared by mixing $0.86 \mathrm{~g}$ of Citric Acid (PM = 210.14 g/mol, C0759 Sigma-Aldrich, St. Louis, MO, USA), $1.74 \mathrm{~g}$ of Sodium Citrate Dihydrate (PM = $294.12 \mathrm{~g} / \mathrm{mol}$, W302600 SigmaAldrich, St. Louis, MO, USA) and $997.4 \mathrm{~g}$ of bi-distilled water with a high degree of purity (ELGA system). Samples of $100 \mathrm{~g}$ of both buffer-based hydrogels were prepared with HT concentrations equal to $0 \mathrm{mM}(0 \mathrm{~g}$ HT), $1 \mathrm{mM}$ (0.0154 g HT), $2 \mathrm{mM}$ (0.0308 $\mathrm{g}$ HT), $5 \mathrm{mM}$ (0.0771 g HT), $10 \mathrm{mM}$ (0.1542 g HT), $50 \mathrm{mM}$ (0.7708 g HT), and $100 \mathrm{mM}$ (1.5416 g HT). Since PEO has been kept constant $(4 \% w / w)$, the grams of Phosphate Buffer Solution or Citrate Buffer Solution to be added in each different solution were calculated accordingly. Mixture of components was prepared with a magnetic stirrer (Joan-SH-2, BIPEE, Shenzen, China) for $0 \mathrm{mM}$ HT hydrogels and with a mechanical stirrer (AM20-D, ArgoLab, Carpi, Italy) for all other hydrogel preparations. Solvation occurred for at least $24 \mathrm{~h}$.

\subsection{Gamma-Ray Irradiation}

The irradiation of HT-based hydrogels was carried out by Gammatom S.r.l. (Guanzate, Italy), at $25 \mathrm{kGy}$ of intensity. Samples were placed in $50 \mathrm{~mL}$ Falcon tubes.

\subsection{Rheological Analysis}

In order to measure the viscosity and to study the rheological properties of the prepared hydrogels, the rheometer RM 200 PLUS (Lamy Rheology, Champagne au Mont d'Or, France) was used. $25 \mathrm{~mL}$ of sample was put into tubes with the corresponding coaxial cylinder measuring systems suitable for the control of homogeneous products, with liquid aspect and with or without particles. RheoTex software PN N311000 (Lamy Rheology, Champagne au Mont d'Or, France) was used for measurements, choosing the "Viscosity" program with the following set-up: pre-shearing time of $10 \mathrm{~s}$ with a shear rate of $10 \mathrm{~s}^{-1}$, 
then the analysis was conducted with a shear rate fixed at $50 \mathrm{~s}^{-1}$ for $60 \mathrm{~s}$; within this time, 100 points have been recorded.

\subsection{IR Chemical Analysis}

In order to perform the chemical analysis of the hydrogels, one drop of each sample was put on aluminum plates at $55^{\circ} \mathrm{C}$ for $24 \mathrm{~h}$ to avoid IR water interference. The FT-IR analysis was performed with the FT-IR spectrometer Spectrum Two (Perkin Elmer, Waltham, MA, USA) equipped with an ATR module, using the dedicated Spectrum 10 Software Suite (Perkin Elmer, Waltham, MA, USA). To analyze chemical changes, an assessment of the areas of peaks of interest has been carried out to obtain a direct measurement of the area under the peaks corresponding to the molar extinction coefficient.

\subsection{HT Extraction and HPLC Quantification}

Hydrogels were sonicated (DK-80, DK Sonic, Shenzen, China) for $30 \mathrm{~min}$ in extraction solvent 70:30 (v/v) ethanol:water to extract HT. Sonicated solution was filtered $(0.22 \mu \mathrm{m})$ before liquid chromatography. The HPLC analysis was performed using Flexar LC (Perkin Elmer, Waltham, MA, USA) equipped with a binary pump and a DAD detector. Chromatographic conditions were: isocratic $70 \%$ ( $0.1 \%$ acetic acid) $30 \%$ (acetonitrile); temperature: $21{ }^{\circ} \mathrm{C}\left( \pm 1{ }^{\circ} \mathrm{C}\right)$; flux and runtime: $1.6 \mathrm{~mL} / \mathrm{min}$ for $10.0 \mathrm{~min}$; absorbance: wavelength of $280 \mathrm{~nm}$ with an analytical bandwidth of $5 \mathrm{~nm}$; injection Volume: $1.0 \mu \mathrm{L}$; column: HC-C18 Agilent $5 \mu \mathrm{m}, 4.6 \times 150 \mathrm{~mm}$.

\subsection{Statistical Analysis}

When applicable, samples were compared with untreated samples (either with HT $0 \mathrm{mM}$ or before irradiation) in the corresponding buffer and differences were tested with Student's $t$-tests.

\section{Results}

\subsection{Effects of Hydroxytyrosol on Rheological Properties of PEO Hydrogels}

Rheological analysis was performed before and after gamma-ray irradiation on all formulations of citrate and phosphate buffer-based PEO $4 \%$ hydrogels, with increasing concentration of HT. HT addition decreases the viscosity of both phosphate and citrate buffer PEO 4\% hydrogels in a concentration-dependent manner (Figure 1a). After gammaray irradiation, hydrogel viscosity highly increases; irradiated formulations with less than $10 \mathrm{mM}$ HT concentration could not be analyzed with the rheometer because of their extreme density. Increasing doses of HT reduces the irradiation-induced viscosity with greater dose-dependent effects on citrate buffer-based hydrogels in comparison to phosphate buffer samples (Figure 1b). 
(a)

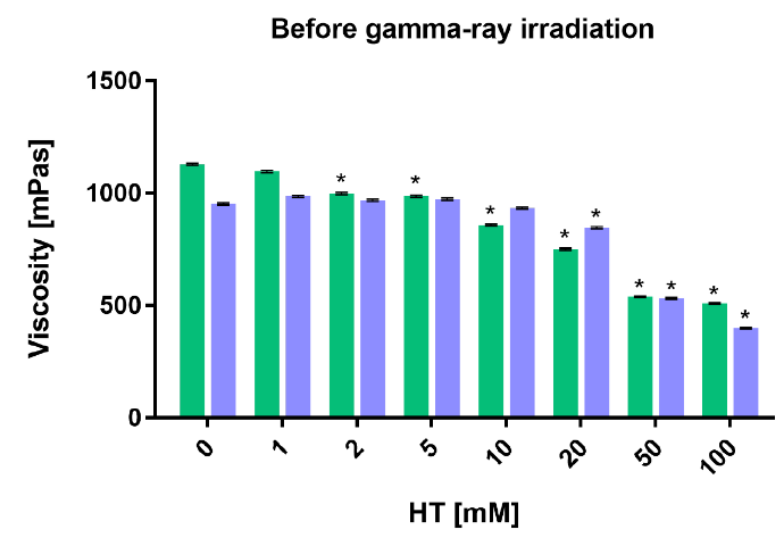

(b)

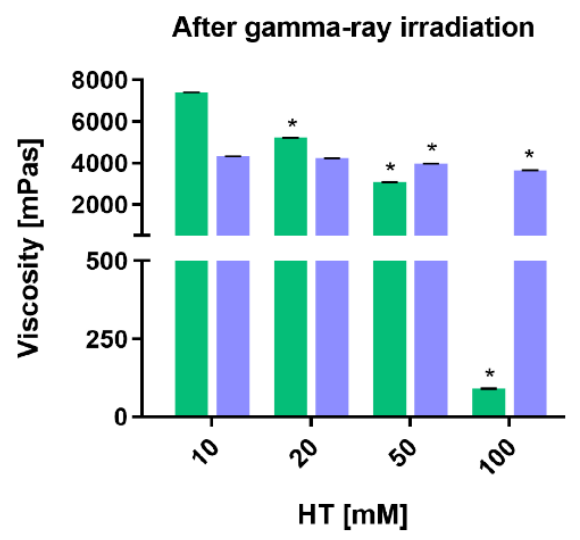

\section{Citrate Buffer Phosphate Buffer}

Figure 1. (a) Change in the viscosity of non-irradiated polyethylene oxide (PEO) $4 \%$ hydrogels when increasing hydroxytyrosol (HT) concentration; ${ }^{*} p<1 \times 10^{-4}$ vs. HT $0 \mathrm{mM}$ samples in the corresponding buffer; (b) Change in the viscosity of PEO $4 \%$ hydrogels when increasing HT concentration after gamma ray irradiation; ${ }^{*} p<1 \times 10^{-4}$ vs. HT $10 \mathrm{mM}$ samples in the corresponding buffer.

3.2. Effects of Citrate and Phosphate Buffer Solutions on the Chemical Structure of Irradiated PEO Hydrogels

IR spectra were analyzed to observe chemical changes of hydrogels after gamma-ray irradiation. Bi-distilled water PEO 4\% hydrogel was used as a control sample to compare the different chemical formulations of phosphate and citrate buffers. Bi-distilled and citrate buffer samples have similar IR spectra before and after irradiation with no evidence of new chemical bond formation. Only an overall reduction in molar extinction coefficients is observable in irradiated samples, indicating a decrease in the vibrational capacity of the chemical bonds (Figure 2a,b). Interestingly, analysis of the fingerprint region of the IR spectrum of the phosphate buffer PEO $4 \%$ hydrogel sample reveals new chemical bond formation showing new peaks at $1260 \mathrm{~cm}^{-1}, 802 \mathrm{~cm}^{-1}$, and $660 \mathrm{~cm}^{-1}$ (Figure 2c).

(a) Bidistilled water PEO 4\% hydrogels

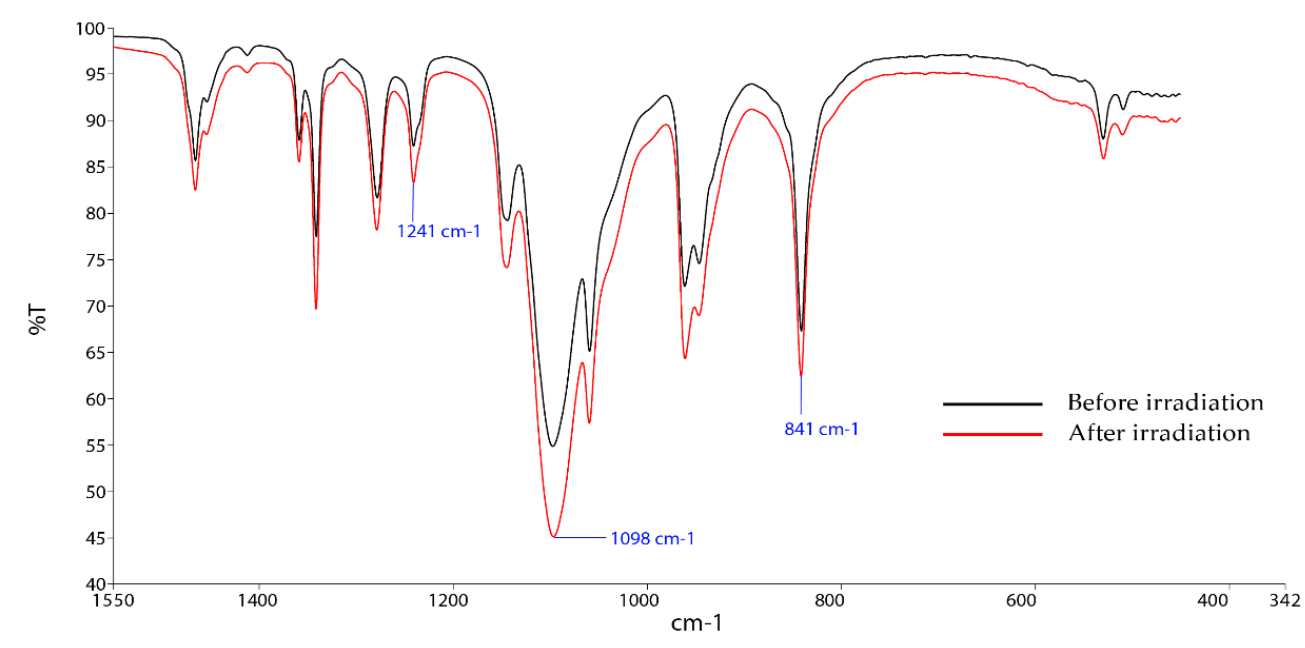

Figure 2. Cont. 
(b)

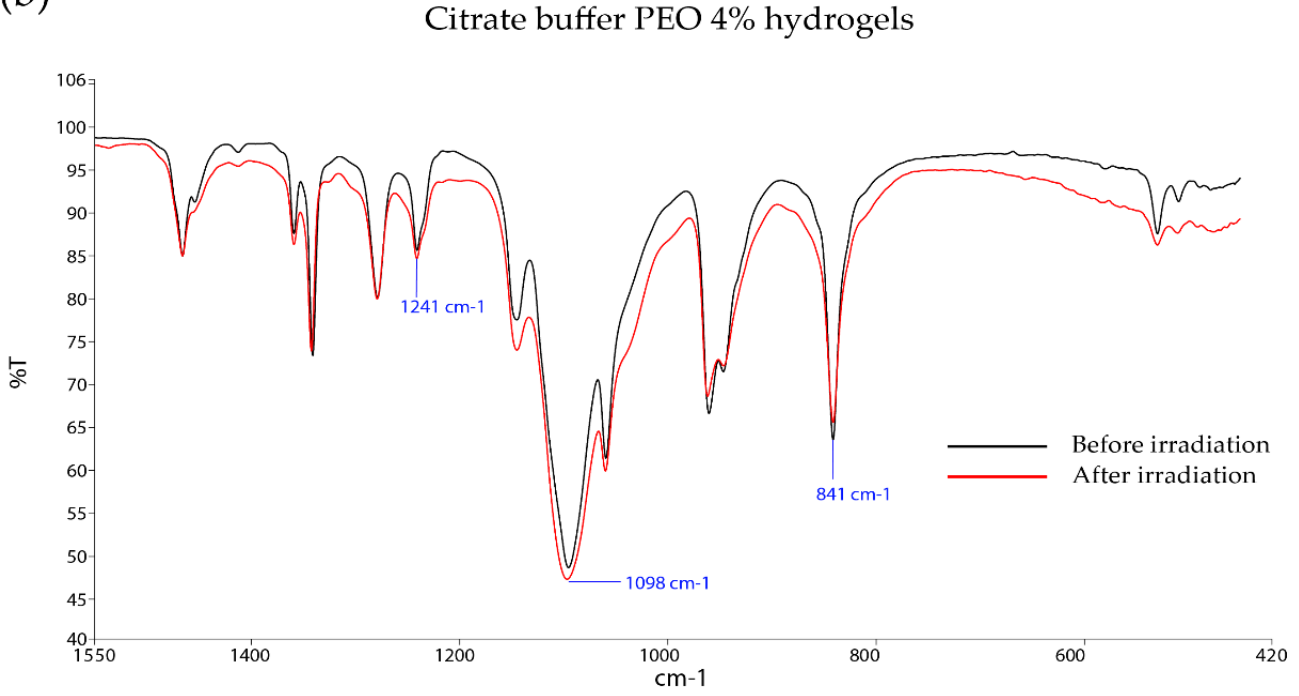

(c)

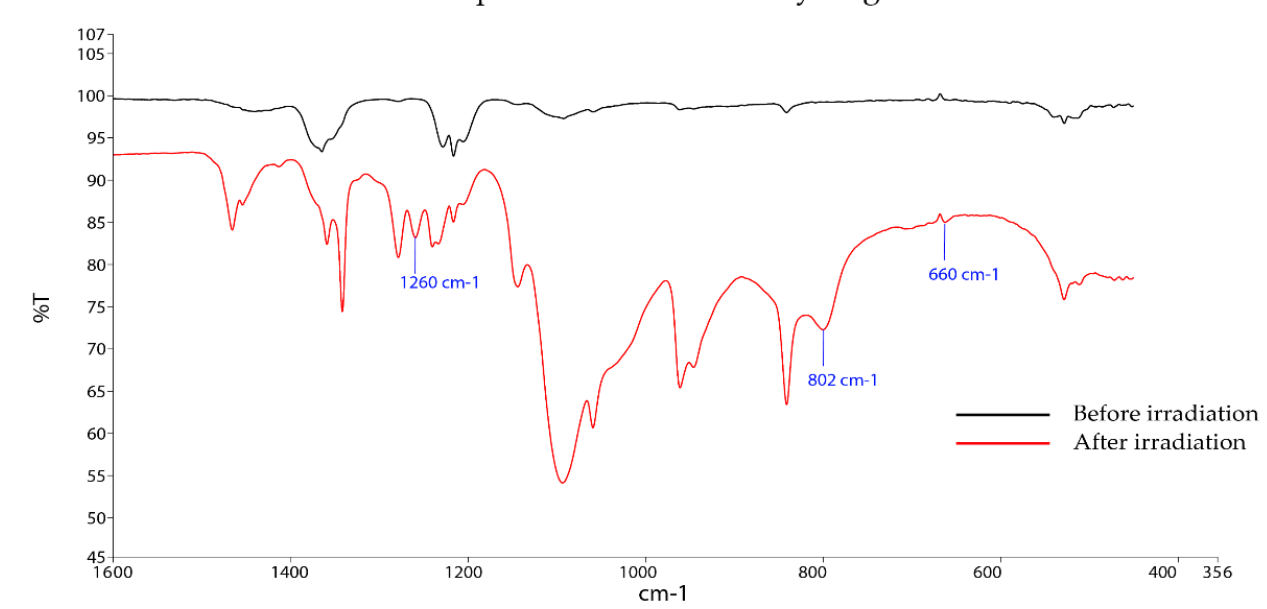

Figure 2. (a) Fingerprint region of IR spectra of bi-distilled water PEO 4\% hydrogels before and after gamma-ray irradiation; (b) Fingerprint region of IR spectra of citrate buffer PEO 4\% hydrogels before and after gamma ray irradiation; (c) Fingerprint region of IR spectra of phosphate buffer PEO 4\% hydrogels before and after gamma-ray irradiation.

\subsection{Hydroxytyrosol Protects PEO Hydrogels from Gamma Ray Irradiation-Induced Polymerization}

The chemical formulations of citrate buffer PEO $4 \%$ hydrogels were analyzed before and after irradiation when increasing HT concentration. Addition of HT increases the vibrational capacity of chemical bonds in both non-irradiated and irradiated samples, although there is a general decrease in the molar extinction coefficients above $20 \mathrm{mM}$ of HT concentration. Irradiation decreases areas of each peaks at $1241 \mathrm{~cm}^{-1}$ (C-O-C stretching), $1098 \mathrm{~cm}^{-1}$ (C-O stretching) and $841 \mathrm{~cm}^{-1}$ (C-O-C stretching) in all hydrogel preparations, as an evidence of stiffening of the polymer structure (Figure 3a). The fold change in the area of the peaks of interest between non-irradiated and irradiated samples decreases with HT concentration higher than $10 \mathrm{mM}$ (Figure 3b). 
(a)
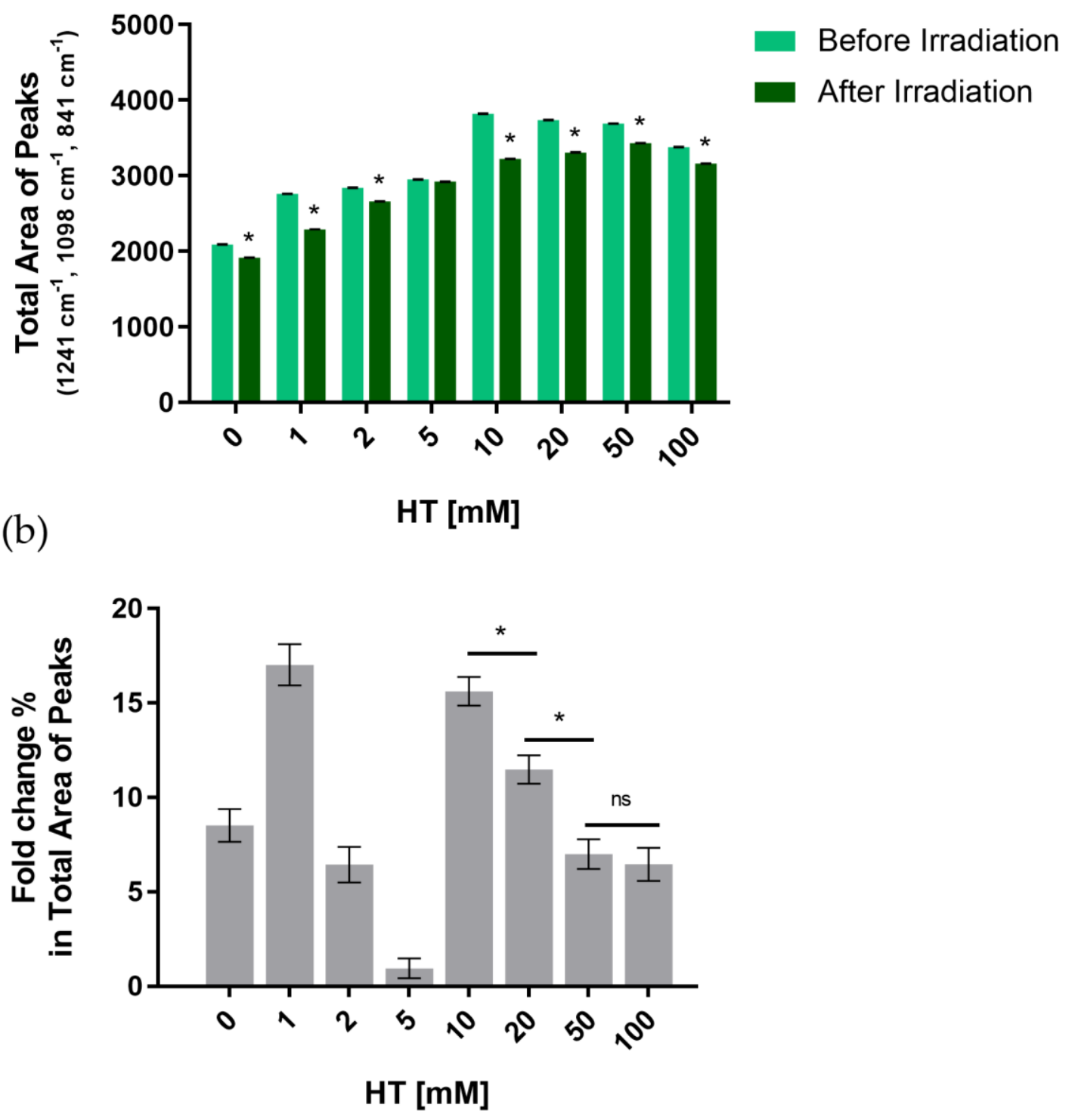

Figure 3. (a) Change in the areas of the peaks of interest (Total Area of Peaks is the sum of areas at $1241 \mathrm{~cm}^{-1}, 1908 \mathrm{~cm}^{-1}$, and $841 \mathrm{~cm}^{-1}$ ) in non-irradiated and irradiated citrate buffer-based PEO hydrogels when increasing HT concentration; ${ }^{*} p<1 \times 10^{-4}$ vs. non-irradiated samples with the same HT concentration; (b) Percentage of fold change in Total Area of Peaks (sum of areas at $1241 \mathrm{~cm}^{-1}, 1908 \mathrm{~cm}^{-1}$, and $841 \mathrm{~cm}^{-1}$ ) between the non-irradiated and irradiated citrate buffer-based PEO hydrogels when increasing HT concentration. ${ }^{*} p<0.01$.

\subsection{Hydroxytyrosol Protects PEO Hydrogels from Gamma Ray Irradiation-Induced Cross-Linking}

The chemical formulations of phosphate buffer PEO 4\% hydrogels were analyzed before and after irradiation when increasing HT concentration. In the phosphate bufferbased hydrogels, the addition of HT prevents the irradiation-induced formation of new chemical bonds $\left(1260 \mathrm{~cm}^{-1}, 802 \mathrm{~cm}^{-1}\right.$, and $\left.660 \mathrm{~cm}^{-1}\right)$ in a dose-dependent manner; above $10 \mathrm{mM}$ of HT there is no evidence of new chemical bond formation (Figure $4 \mathrm{a}$ ). 


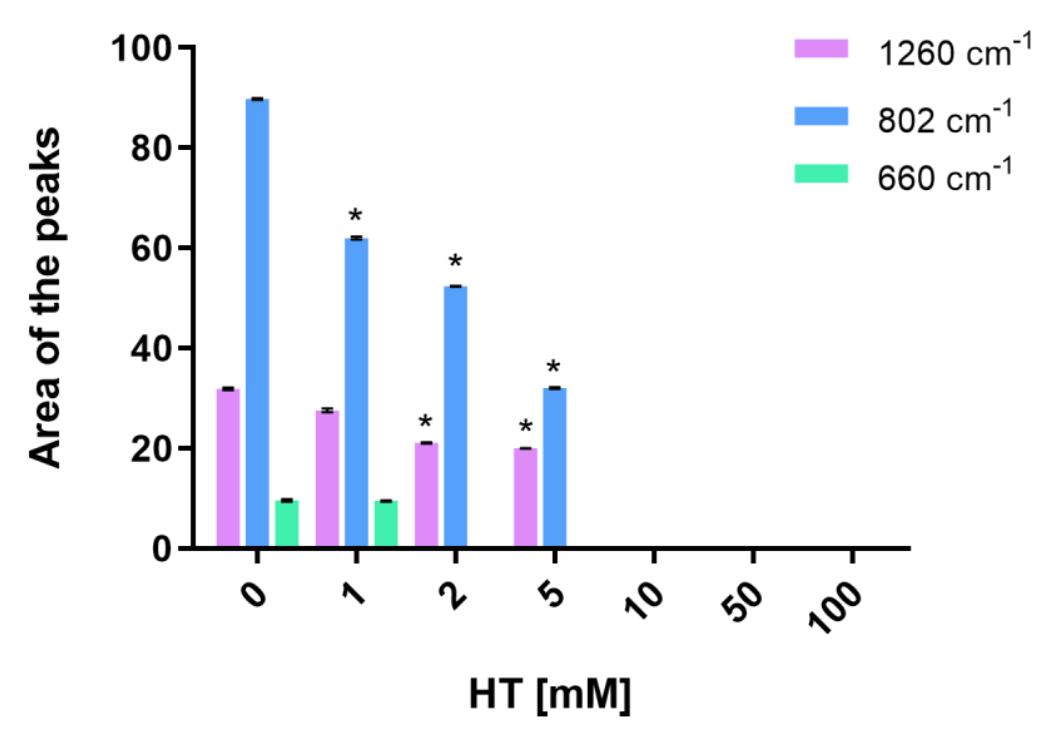

Figure 4. Change in the areas of the peaks of interest $\left(1260 \mathrm{~cm}^{-1}, 802 \mathrm{~cm}^{-1}\right.$, and $\left.660 \mathrm{~cm}^{-1}\right)$ in irradiated phosphate buffer-based PEO hydrogels when increasing HT concentration; ${ }^{*} p<1 \times 10^{-4}$ vs. HT 0 mM samples.

\subsection{Quantification of Residual Hydroxytyrosol in PEO Hydrogels after Sterilization by Gamma Irradiation}

HPLC quantification of residual HT in irradiated hydrogels was performed to test the consumption of HT after gamma-ray-induced oxidation. Averagely, HT concentrations are highly preserved in irradiated citrate buffer hydrogels in comparison with the notirradiated samples, showing only little consumption of the molecule. On the other hand, HT concentrations are almost halved after irradiation in phosphate buffer hydrogel formulations (Table 1), probably due to the HT consumption exerting its anti-oxidative activity.

Table 1. High-performance liquid chromatography (HPLC) quantification of residual HT in phosphate and citrated buffer PEO 4\% hydrogels after gamma-ray irradiation.

\begin{tabular}{|c|c|c|c|c|}
\hline \multirow{2}{*}{$\begin{array}{l}\text { HT Starting } \\
\text { Concentration }\end{array}$} & \multicolumn{2}{|c|}{$\begin{array}{l}\text { Citrate Buffer-Based Polyethylen Oxide } \\
\text { (PEO) Hydrogel }\end{array}$} & \multicolumn{2}{|c|}{ Phosphate Buffer-Based PEO Hydrogel } \\
\hline & $\begin{array}{c}(\mathrm{HT}) \\
\text { Before Irradiation }\end{array}$ & $\begin{array}{c}\text { (HT) } \\
\text { After Irradiation }\end{array}$ & $\begin{array}{c}(\mathrm{HT}) \\
\text { Before Irradiation }\end{array}$ & $\begin{array}{c}{[\mathrm{HT}]} \\
\text { After Irradiation }\end{array}$ \\
\hline $1 \mathrm{mM}$ & 0.999 & / & 0.849 & / \\
\hline $2 \mathrm{mM}$ & 1.823 & / & 1.734 & / \\
\hline $5 \mathrm{mM}$ & 4.835 & 4.301 & 4.785 & / \\
\hline $10 \mathrm{mM}$ & 9.867 & 9.411 & 8.913 & 5.761 \\
\hline $20 \mathrm{mM}$ & 19.852 & 19.432 & 17.881 & 11.327 \\
\hline $50 \mathrm{mM}$ & 49.926 & 48.966 & 42.859 & 28.059 \\
\hline $100 \mathrm{mM}$ & 99.918 & 98.998 & 91.875 & 56.107 \\
\hline
\end{tabular}

\section{Discussion}

We found that the addition of HT in PEO hydrogels can prevent cross-linking phenomena and the increase of viscosity caused by gamma-ray irradiation. Gamma-rays induce the creation of radical species interacting with water $(\mathrm{H} \bullet, \mathrm{OH} \bullet)$, buffer components (ROO•), and polymers. We hypothesized that free radicals could break the polymer chains, thus enhancing their reactivity and either triggering interchain polymerization (Figure 5a), intrachain cross-linking (Figure 5b), or cross-linking with phosphate (Figure 5c). The proposed underlying mechanism is likely to be the following: in hydrogels, HT exerts anti-oxidant activity by scavenging free radicals through proton release and structural conjugation $[7,8]$. The free radical scavenging activity, which is possible for the highly reactive structure 
of $o$-quinone derived from HT (Scheme 1), limits or even prevents polymerization and cross-linking phenomena.

\section{Polymerization}

(a)<smiles>COCCOC</smiles>

Cross-linking

(b)<smiles>COCCOCCOC(C)C(C)OC</smiles>

(c)

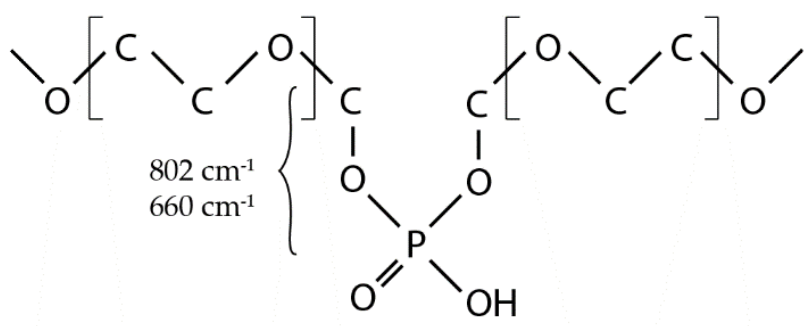

Figure 5. Hypothesis for the irradiation-induced reactions occurring in PEO hydrogels that are prevented or limited by the free radical scavenging activity of HT. (a) Interchain polymerization reaction suggested by the decrease in the areas of peaks of at $1241 \mathrm{~cm}^{-1}, 1908 \mathrm{~cm}^{-1}$, and $841 \mathrm{~cm}^{-1}$ observed in IR spectra in all irradiated buffer; (b) Intrachain cross-linking reaction suggested by the new peak at $1260 \mathrm{~cm}^{-1}$ observed in IR spectra in irradiated phosphate-based hydrogels; (c) Cross-linking reaction between PEO chains and phosphate suggested by the new peaks at $802 \mathrm{~cm}^{-1}$ and $660 \mathrm{~cm}^{-1}$ observed in IR spectra in irradiated phosphate-based hydrogels.

This protective effect is concentration-dependent; moreover, it maintains the rheological properties of hydrogels even after sterilization, being suitable for the production of medical devices. Indeed, hydrogels have different clinical applications that rely on their mechanical properties [1,2]; thus, these structural characteristics should not be altered in order to preserve the benefits of biocompatibility, scaffolding, or injectability.

The cross-linking phenomenon causes embrittlement and stiffness in hydrogels which consequently lose their mechanical properties. This was observed in IR analysis of phosphate buffer-based PEO hydrogels where irradiation of the polymer structure generates free radicals that rearrange C-O-C chemical bonds $\left(1260 \mathrm{~cm}^{-1}\right)$ between the polymeric chains. Moreover, new phosphate bonds formation was observed at $802 \mathrm{~cm}^{-1}$ and $660 \mathrm{~cm}^{-1}$, corresponding to the C-O-P stretching and scissoring [12]. The chemical structure of HT can stop the chain propagation reactions by scavenging the free radicals $[7,8,13]$. Even if HT can prevent cross-linking reactions, these chemical changes suggest that other buffer solutions rather than phosphate buffer may be preferred for the production of medical-grade hydrogels to avoid any biological unpredictable interaction. Indeed, citrate buffer-based hydrogels are more stable according to the results obtained. One reason could be that citrate buffer stabilizes $\mathrm{pH}$ condition for HT molecules without altering hydrogel mechanical properties, as confirmed by rheological analysis. In IR analysis of citrate buffer-based hydrogels, only an overall decrease in the molar extinction coefficients is observed, suggesting a polymerization reaction between chains. HT can prevent polymerization by exerting its free radical scavenger activity, as shown by HT concentration higher than $10 \mathrm{mM}$. However, HT addition also decreases the viscosity and influences the vibrational capacity of the chemical bonds in non-irradiated samples, probably because HT surfactant properties may help the polymer hydration, therefore its solubilization [14]. Because of this activity, protection from polymerization reactions is not fully measurable in this ex- 
perimental setting. However, rheological analysis shows that the presence of HT in citrate buffer-based hydrogels dramatically lowers the irradiation-induced increase in viscosity in a dose-dependent manner.

Other molecules, such as Vitamin C [4] or Vitamin E [5] have been explored as antioxidant agents in hydrogels preparation, but even if HT is a superior anti-oxidant and radical scavenger than Vitamin C [6], few data exist on this topic. HT is a remarkable molecule showing a broad spectrum of biological properties, e.g., anti-cancer, anti-microbial, antiinflammatory, and anti-viral activities $[7,10,13]$, which can be exploited in tissue engineering and medical application strategies. Our HPLC analysis shows that more than 50\% of HT concentration is preserved after preventing cross-linking reactions in irradiated phosphate buffer-based PEO hydrogels, and HT is almost totally preserved in citrate buffer-based PEO hydrogels. The anti-microbial properties of HT are helpful against microbial agents typical of periodontal diseases [15] or infected chronic skin wounds [16], thus making the molecule an ideal ingredient for the preparation of medical-grade hydrogels.

A limitation of this first study is the impossibility of testing the viscosity of all hydrogel formulations within this experimental setting. The adopted rheometer cannot test semisolid hydrogels obtained after irradiation in the absence of or with minimal amount of HT. Future characterization will involve the use of texture analyzer, i.e., relaxation tests with penetrating probes to provide information on the consistency, viscosity, and elasticity of all hydrogel formulations [17]. Moreover, future analyses can include irradiation-induced effects on swelling and gelation rate of hydrogels.

In conclusions, our findings suggest a novel exploitation of HT free radical scavenging activity to prevent irradiation-induced cross-linking reactions, thus helping the production of medical-grade polymeric hydrogels with optimized viscosity and injectability.

Author Contributions: Conceptualization, P.F. and M.F.; methodology, P.F. and M.F.; software, P.F.; validation, P.F.; formal analysis, P.F., M.F., and V.C.; resources, P.F. and M.F.; data curation, P.F., M.F., V.C., and O.S.; writing—original draft preparation, O.S.; writing—review and editing, P.F., M.F., and O.S.; visualization, P.F., M.F., and L.S.; supervision, P.F. and L.S.; project administration, P.F. and M.F.; funding acquisition, P.F. and M.F. All authors have read and agreed to the published version of the manuscript.

Funding: This research received no external funding.

Institutional Review Board Statement: Not applicable.

Informed Consent Statement: Not applicable.

Data Availability Statement: The data presented in this study are available on request from the corresponding author. The data are not publicly available due to confidentiality.

Conflicts of Interest: The authors declare no conflict of interest.

\section{References}

1. Pal, K.; Banthia, A.K.; Majumdar, D.K. Polymeric hydrogels: Characterization and biomedical applications. Des. Monomers Polym. 2009, 12, 197-220. [CrossRef]

2. Pina, S.; Ribeiro, V.P.; Marques, C.F.; Maia, F.R.; Silva, T.H.; Reis, R.L.; Oliveira, J.M. Regenerative Medicine Applications. Materials 2019, 12, 1824. [CrossRef] [PubMed]

3. Dai, Z.; Ronholm, J.; Tian, Y.; Sethi, B.; Cao, X. Sterilization techniques for biodegradable scaffolds in tissue engineering applications. J. Tissue Eng. 2016, 7, 1-13. [CrossRef] [PubMed]

4. Oral, E.; Bodugoz-Senturk, H.; Macias, C.; Muratoglu, O.K. Vitamin C hinders radiation cross-linking in aqueous poly(vinyl alcohol) solutions. Nucl. Instrum. Methods Phys. Res. Sect. B Beam Interact. Mater. Atoms 2007, 265, 92-97. [CrossRef]

5. Oral, E.; Ghali, B.W.; Rowell, S.L.; Micheli, B.R.; Lozynsky, A.J.; Muratoglu, O.K. A surface crosslinked UHMWPE stabilized by vitamin E with low wear and high fatigue strength. Biomaterials 2010, 31, 7051-7060. [CrossRef] [PubMed]

6. Fruhwirth, G.O.; Wagner, F.S.; Hermetter, A. The $\alpha$ pROX assay: Fluorescence screening of the inhibitory effects of hydrophilic antioxidants on protein oxidation. Anal. Bioanal. Chem. 2006, 384, 703-712. [CrossRef] [PubMed]

7. Fernández-Bolaños, J.G.; López, Ó.; López-García, M.Á.; Marset, A. Biological Properties of Hydroxytyrosol and Its Derivatives. In Olive Oil-Constituents, Quality, Health Properties and Bioconversions; InTech: London, UK, 2012; pp. 375-396. ISBN 978-953-307-921-9. 
8. Umeno, A.; Takashima, M.; Murotomi, K.; Nakajima, Y.; Koike, T.; Matsuo, T.; Yoshida, Y. Radical-scavenging activity and antioxidative effects of olive leaf components oleuropein and hydroxytyrosol in comparison with homovanillic alcohol. J. Oleo Sci. 2015, 64, 793-800. [CrossRef] [PubMed]

9. Yüksel Aydar, A.; Öncü Öner, T.; Üçok, E.F.; Aydar, A.Y.; Assistant, R. Effects of Hydroxytyrosol on Human Health. EC Nutr. 2017, 11, 147-157.

10. Granados-Principal, S.; Quiles, J.L.; Ramirez-Tortosa, C.L.; Sanchez-Rovira, P.; Ramirez-Tortosa, M.C. Hydroxytyrosol: From laboratory investigations to future clinical trials. Nutr. Rev. 2010, 68, 191-206. [CrossRef] [PubMed]

11. Robles-Almazan, M.; Pulido-Moran, M.; Moreno-Fernandez, J.; Ramirez-Tortosa, C.; Rodriguez-Garcia, C.; Quiles, J.L.; RamirezTortosa, M. Hydroxytyrosol: Bioavailability, toxicity, and clinical applications. Food Res. Int. 2018, 105, 654-667. [CrossRef]

12. Davidson, G. Spectroscopic Properties of Inorganic and Organometallic Compounds: Volume 26; Royal Society of Chemistry: Cambridge, UK, 1993; ISBN 978-0-85186-474-7.

13. Tuck, K.L.; Hayball, P.J. Major phenolic compounds in olive oil: Metabolism and health effects. J. Nutr. Biochem. 2002, 13, 636-644. [CrossRef]

14. Martínez, L.; Ros, G.; Nieto, G. Hydroxytyrosol: Health Benefits and Use as Functional Ingredient in Meat. Medicines 2018, 5, 13. [CrossRef] [PubMed]

15. Matsumoto, G. Periodontal Regeneration Using Gelatin Hydrogels Incorporating Basic Fibroblast Growth Factor. Biomed. J. Sci. Tech. Res. 2018, 4, 3820-3824. [CrossRef]

16. Da Silva, L.P.; Reis, R.L.; Correlo, V.M.; Marques, A.P. Hydrogel-Based Strategies to Advance Therapies for Chronic Skin Wounds. Annu. Rev. Biomed. Eng. 2019, 21, 145-169. [CrossRef] [PubMed]

17. Mei, X.; Etzler, F.M.; Wang, Z. Use of texture analysis to study hydrophilic solvent effects on the mechanical properties of hard gelatin capsules. Int. J. Pharm. 2006, 324, 128-135. [CrossRef] [PubMed] 